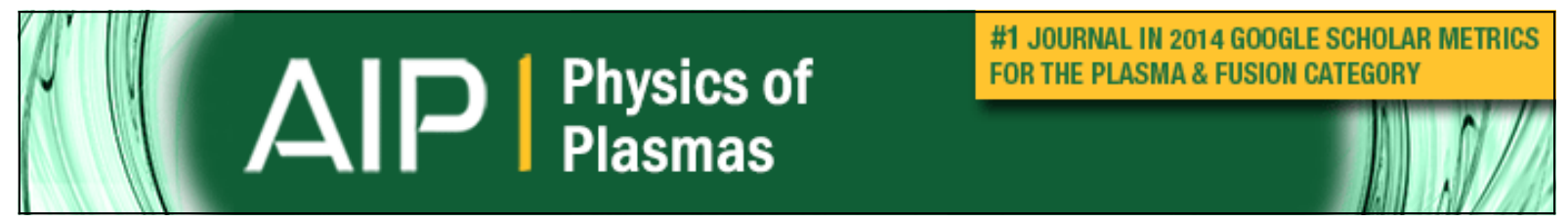

\title{
Turbulent edge structure formation in complex configurations
}

\author{
A. Kendl, B. D. Scott, R. Ball, and R. L. Dewar
}

Citation: Physics of Plasmas 10, 3684 (2003); doi: 10.1063/1.1602074

View online: http://dx.doi.org/10.1063/1.1602074

View Table of Contents: http://scitation.aip.org/content/aip/journal/pop/10/9?ver=pdfcov

Published by the AIP Publishing

\section{Articles you may be interested in}

Electromagnetic turbulent structures: A ubiquitous feature of the edge region of toroidal plasma configurations Phys. Plasmas 22, 012310 (2015); 10.1063/1.4906869

Reducing turbulent transport in toroidal configurations via shapinga)

Phys. Plasmas 18, 056101 (2011); 10.1063/1.3560591

Coupled drift-wave-zonal flow model of turbulent transport in the tokamak edge

Phys. Plasmas 12, 092307 (2005); 10.1063/1.2010473

Drift wave versus interchange turbulence in tokamak geometry: Linear versus nonlinear mode structure

Phys. Plasmas 12, 062314 (2005); 10.1063/1.1917866

Low to high confinement transition theory of finite-beta drift-wave driven shear flow and its comparison with data from DIII-D

Phys. Plasmas 11, 1109 (2004); 10.1063/1.1638381

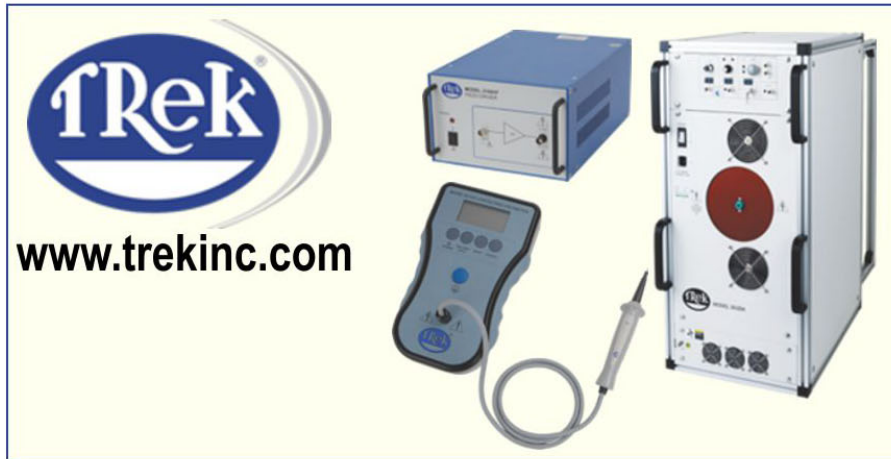

HIGH-VOLTAGE AMPLIFIERS AND ELECTROSTATIC VOLTMETERS

ENABLING RESEARCH AND INNOVATION IN DIELECTRICS, MICROFLUIDICS, MATERIALS, PLASMAS AND PIEZOS 


\title{
Turbulent edge structure formation in complex configurations
}

\author{
A. Kendl and B. D. Scott \\ Max-Planck-Institut für Plasmaphysik, EURATOM Association, D-85748 Garching, Germany \\ R. Ball and R. L. Dewar \\ Australian National University, Department of Theoretical Physics, Canberra ACT 0200, Australia
}

(Received 10 June 2003; accepted 25 June 2003)

\begin{abstract}
Formation of nonlinear structures in drift-Alfvén turbulence is investigated in the often complex edge geometries of stellarator and tokamak configurations, by analysis of drift wave turbulence simulations using a model in which three-dimensional magnetic geometries are approximated. The structures of parallel mode extension, radially sheared zonal flows and perpendicular mode spectra are highlighted in particular for three-dimensional stellarator magnetic fields and shaped tokamaks. Specific characteristics of advanced stellarators in comparison to (lower aspect ratio) circular tokamaks are a less pronounced ballooning structure of the modes, a strong influence of local magnetic shear on amplitude structure and average, and stronger level of zonal flows due to lower geodesic curvature. (C) 2003 American Institute of Physics. [DOI: 10.1063/1.1602074]
\end{abstract}

\section{INTRODUCTION}

In magnetic confinement devices the plasma edge region is typically dominated by turbulent density, temperature, and potential fluctuations that give rise to anomalous transport fluxes. Experiments have shown the fluctuations to have amplitudes and phase relationships that are similar in stellarators and tokamaks, and anisotropic correlation lengths that are typical of drift-wave turbulence. These similarities in the structure and dynamics of edge turbulence and diffusion across devices are reviewed in Refs. 1 and 2. Such universal character should not be surprising, because the fundamental mechanisms of drift wave instability ${ }^{3,4}$ are independent of confinement geometry. Gradients of density and electron and ion temperature provide a source of free energy that may be tapped by dissipative processes and Alfvén wave dynamics. Catalytic and damping effects, on the other hand, are due to field line curvature, magnetic and velocity shear, and differ among specific geometries.

More recent studies involving simulations of drift waves in shaped magnetic fields show considerable influence of magnetic field geometry on instability and transport. ${ }^{5,6}$ Field line shaping in a tokamak is usually due to secondary properties like, for example, elongation of the plasma torus, but nontrivial geometry is an endemic feature of stellarators. Insights into the dynamics of the turbulence and the underlying processes coupling the variations in the background magnetic field with the plasma fluctuations can be gained by comparative computational analysis of different configurations.

In our present work we analyze simulations of edge turbulence structures in the geometries of the Helias stellarator Wendelstein $7-\mathrm{X}^{7}$ the Heliac $\mathrm{H} 1-\mathrm{NF}^{8}$ and a simple tokamak model. The drift-Alfvén model we employ and some characteristics of plasma edge turbulence are described in Sec. II. In Sec. III exemplar settings and parameters for the turbulence code and computational requirements are given, and the representation of magnetic field geometries in the code is discussed. The two stellarators and their flux tube representation are described in Sec. IV. In Sec. V we give a brief overview of turbulence structures in the plasma edge, then present and discuss the results on simulated structures in parallel, radial, and perpendicular directions. Some of the specific characteristics of these stellarators in comparison to (lower aspect ratio) circular tokamaks are a less pronounced ballooning structure of the modes, a strong influence of local magnetic shear on amplitude structure and average, and stronger level of zonal flows due to lower geodesic curvature. It is also found that the typical signatures of drift wave turbulence are retained across different configurations, but the consequent transport levels depend on magnetic field geometry. A brief wrap-up and the conclusions from this study are given in Sec. VI.

\section{DRIFT-ALFVÉN EQUATIONS AND CODE}

The turbulence simulations were performed using the parallel code DALF3, ${ }^{9}$ which uses the four-field version of the dissipative drift-Alfvén model, ${ }^{10}$ with amplitude fluctuations of the electrostatic potential $\widetilde{\phi}$, electron pressure $\widetilde{p}_{e}$, and disturbances of the parallel current $\widetilde{J}_{\|}$, and parallel ion velocity $\widetilde{u}_{\|}$as dependent variables. The equations are derived under gyro/drift ordering, ${ }^{3,4,11}$ in a three-dimensional globally consistent flux tube geometry, ${ }^{12,13}$ and appear as

$$
\begin{aligned}
& \frac{n M_{i} c^{2}}{B^{2}} \frac{d}{d t} \widetilde{\Omega}=\nabla_{\|} \widetilde{j}_{\|}-\mathcal{K}\left(\widetilde{p}_{e}\right), \\
& \frac{1}{c} \frac{\partial}{\partial t} \widetilde{A}_{\|}+\frac{m_{e}}{e} \frac{d}{d t} \widetilde{j}_{\|}=\frac{1}{n e} \nabla_{\|}\left(p_{e}+\widetilde{p}_{e}\right)-\nabla_{\|} \widetilde{\phi}-\eta_{\|} \widetilde{j}_{\|}, \\
& \frac{d}{d t}\left(p_{e}+\widetilde{p}_{e}\right)=\frac{T_{e}}{e} \nabla_{\|} \widetilde{j}_{\|}-p_{e} \nabla_{\|} \widetilde{u}_{\|}+p_{e} \mathcal{K}(\widetilde{\phi})-\frac{T_{e}}{e} \mathcal{K}\left(\widetilde{p}_{e}\right), \\
& n M_{i} \frac{d}{d t} \widetilde{u}_{\|}=-\nabla_{\|}\left(p_{e}+\widetilde{p}_{e}\right),
\end{aligned}
$$


with the parallel magnetic potential $\widetilde{A}_{\|}$given by

$$
\tilde{j}_{\|}=-\frac{c}{4 \pi} \nabla_{\perp}^{2} A_{\|},
$$

through Ampère's law, and the vorticity

$$
\widetilde{\Omega}=\nabla_{\perp}^{2} \widetilde{\phi},
$$

where $\eta_{\|}$is the parallel resistivity, ${ }^{14} m_{e}$ and $M_{i}$ are the electron and ion masses, $n$ is the electron (and ion) density, and $T_{e}$ is the electron temperature with pressure $p_{e}=n T_{e}$. Action of $d_{t}$ and $\nabla_{\|}$upon $p_{e}$ represents the background gradient drive terms. The differential operators are the advective derivative $d_{t}=\partial_{t}+\mathbf{v}_{E} \cdot \nabla$, the parallel gradient $\nabla_{\|}=(1 / B)(\mathbf{B}$ $\left.+\widetilde{\mathbf{B}}_{\perp}\right) \cdot \nabla$, the perpendicular Laplacian $\nabla_{\perp}^{2}=\nabla \cdot\left[\left(-1 / B^{2}\right) \mathbf{B}\right.$ $\times(\mathbf{B} \times \nabla)]$, and the curvature operator $\mathcal{K}=\nabla \cdot\left[\left(c / B^{2}\right) \mathbf{B}\right.$ $\times \nabla)]$, in which the $\mathbf{E} \times \mathbf{B}$ velocity, $\mathbf{v}_{E}=\left(c / B^{2}\right) \mathbf{B} \times \nabla \widetilde{\phi}$, and the magnetic field disturbances, $\widetilde{\mathbf{B}}_{\perp}=(-1 / B) \mathbf{B} \times \nabla \widetilde{A}_{\|}$, provide the nonlinearities. This constitutes the most basic model containing the principal interactions of dissipative drift wave physics in a general closed magnetic flux surface geometry. The drift wave coupling effect is described by $\nabla_{\|}$acting upon $\widetilde{p}_{e} / p_{e}-e \widetilde{\phi} / T_{e}$ and $\widetilde{J}_{\|}$, while interchange forcing is described by $\mathcal{K}$ acting upon $\widetilde{p}_{e}$ and $\widetilde{\phi} .{ }^{10}$ In the cases we are studying, the drift wave effect is qualitatively more important, ${ }^{9}$ while the most important role for $\mathcal{K}$ is to regulate the zonal flows. ${ }^{15}$ Local shear is described by the entrance of the metric elements into $\nabla_{\perp}^{2}$, and also the spatial dependence of the coupling of drift planes by $\nabla_{\|} \cdot{ }^{13}$

The electromagnetic drift wave turbulence described by this set of equations is basically nonlinear, nonperiodic motion involving disturbances on a background thermal gradient of a magnetized plasma and eddies of fluid like motion in which the advecting velocity of all charged species is the $\mathbf{E}$ $\times \mathbf{B}$ velocity. Note that in this model pressure and density are considered interchangeably, i.e., we consider situations of equal steepness in all the gradients, where the dynamics of the temperatures introduces no new qualitative effects (compare Ref. 9). The disturbances in the electric field $\mathbf{E}$ implied by the presence of these eddies are caused by the tendency of the electron dynamics to establish a force balance along the magnetic field $\mathbf{B}$. Pressure disturbances have their parallel gradients balanced by a parallel electric field, whose static part is given by the parallel gradient of the electrostatic potential. This potential in turn is the stream function for the $\mathbf{E} \times \mathbf{B}$ velocity in drift planes, which are locally perpendicular to the magnetic field. The turbulence is driven by the background gradient, and the electron pressure and electrostatic potential are coupled together through parallel currents. Departures from the static force balance are mediated primarily through electromagnetic induction and resistive friction, but also the electron inertia, which is not negligible.

The advection velocity is purely the $\mathbf{E} \times \mathbf{B}$ velocity because of well-known diamagnetic cancellation effects. The diamagnetic velocity is due to the fact that in a pressure gradient particles on gyro-orbits about gyrocenters displaced in a direction along the pressure gradient, but whose actual particle orbits meet tangentially in between, will meet with differing numbers due to the density part of the gradient, and differing velocities due to the temperature part of the gradient. The net difference is the diamagnetic flux (or current). But in a homogeneous magnetic field the gyrocenters do not move, and so there is no transport. Similar cancellations involve the diamagnetic heat flux in the temperature gradient ${ }^{16}$ and the diamagnetic momentum flux, also called the gyroviscosity, in the momentum equation. ${ }^{11}$ In an inhomogeneous magnetic field (in which $\nabla B$ and curvature drifts are present), the diamagnetic fluxes enter only as quasistatic compressional effects, commonly referred to as curvature terms. The net effect is that the diamagnetic velocity always cancels out of the $\mathbf{v} \cdot \nabla$ terms, and as such does not participate in actual transport. This is much more explicit in the gyrofluid model, ${ }^{17}$ in which the $\nabla B$ and curvature terms enter only when taking moments over the $\nabla B$ and curvature drift terms; i.e., here as well there is no direct participation in advection by these terms.

\section{COMPUTATIONAL AND GEOMETRIC ISSUES}

\section{A. Code parameters and computational requirements}

The toroidal geometry is represented by a globally consistent flux tube with local coordinates $(x, y, z)$, where all background parameters (thermal gradients as well as the geometry) are fixed over the poloidal $(x, y)$ region. The computational domain is set to $64 \times 256$ nodes in units of the drift scale

$$
\rho_{s}=(c / e B) \sqrt{T_{e} M_{i}},
$$

for $(x, y)$ and 16 nodes in one field line connection length $(2 \pi q R)$ in $-\pi<z<\pi$. Boundary conditions are periodic in $y$ and Dirichlet in $x$. Nominal values for the parameters were chosen to reflect typical tokamak and stellarator edge conditions: Collisionality $C=\left(n e^{2} \eta_{\|} / T_{e}\right)\left(c_{s} / L_{\perp}\right)\left(q^{2} R^{2}\right)=4$, magnetic induction $\hat{\beta}=\epsilon\left(4 \pi p_{e} / B^{2}\right)=1$, electron inertia $\hat{\mu}$ $=\epsilon\left(m_{e} / M_{i}\right)=5$, and ion inertia $\epsilon=\left(q R / L_{\perp}\right)^{2}=18350$, where $L_{\perp}$ is the background gradient scale length for $p_{e}$, and $c_{s}=\sqrt{T_{e} / M_{i}}$ is the sound speed. The computations are initialized through a pressure disturbance amplitude well above unity which is driven nonlinearly and then relaxes to a steady turbulent state (compare Refs. 4 and 9). The units of amplitude and transport flux are the standard gyro-Bohm ones, $\left(\rho_{s} / L_{\perp}\right)$, and $p_{e} c_{s}\left(\rho_{s} / L_{\perp}\right)^{2}$, respectively.

Spatial and temporal scales in the computations have to be chosen such that all turbulent structures are well resolved. The radial domain size should reflect the typical edge steep gradient region extension. As a reasonable and representative choice $n_{x}=64$ nodes of size $\rho_{s}$ are used. The anisotropy of turbulent structures requires a larger extension in $y$. The domain size aspect ratio has to be increased until results converge, for which $L_{y} / L_{x}=4$ is a safe choice, and thus $n_{y}$ $=256$.

The time step is set to $\Delta t=0.05$. The observed emergence of structures from an initially randomly disturbed state on the way to fully developed turbulence requires sufficiently long simulation times. Depending on the parameters and geometry, saturation can be reached quickly, after $t$ $>200$, or slowly, only after $t>5000$. Assertions on final 


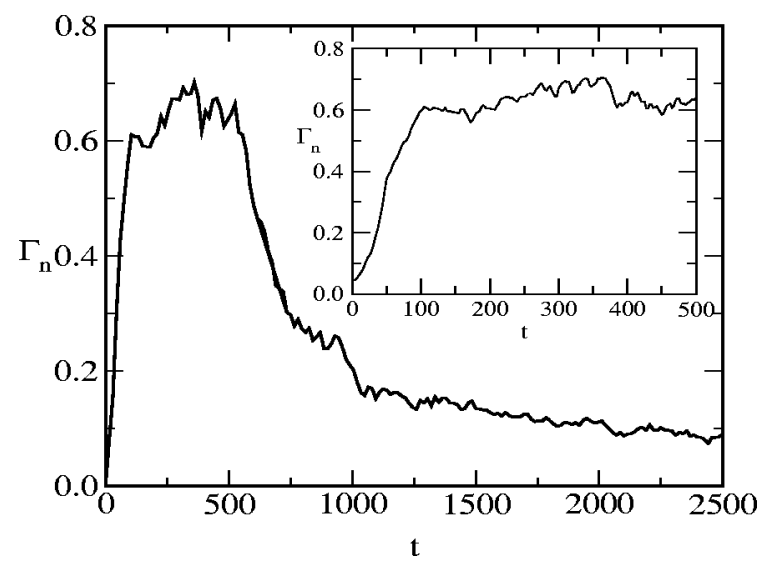

FIG. 1. Time evolution of turbulent transport in a sheared slab model: the emergence of zonal flow structures occurs after an initially quiescent phase after $t \approx 500$. Computations that do not take into acccount the long time behavior and are stopped too early (like in the inset graph) may mislead to erroneous conclusions.

transport and turbulence levels are based on time averages over the saturated phase only. These levels will of course also fluctuate around a mean value during the saturated phase and may exhibit more or less violent "intermittent" deviations from this mean.

We demonstrate the necessity for long simulation times with an example in sheared slab geometry for $\mathcal{K}=0$ and $\hat{s}$ $=1.5$ : Following the time evolution of $\Gamma_{n}(t)$ as long as $t$ $=500$, as displayed in the inset graph in Fig. 1, we get the impression of saturation after around $t>200$. However, if we take the computation to much longer times, drastic changes occur in the transport level: The slow emergence of strong zonal flow structures gradually suppresses the turbulence to low amplitudes, that reach final saturation only after $t$ $>2000$. Conclusions based on simulations stopped after the first seemingly saturated phase with quiescent flow dynamics may lead to erroneous results as illustrated in Fig. 2: The magnetic shear dependence of transport, $\Gamma_{n}(\hat{s})$, obtained from such underdeveloped states would show an inverse be-

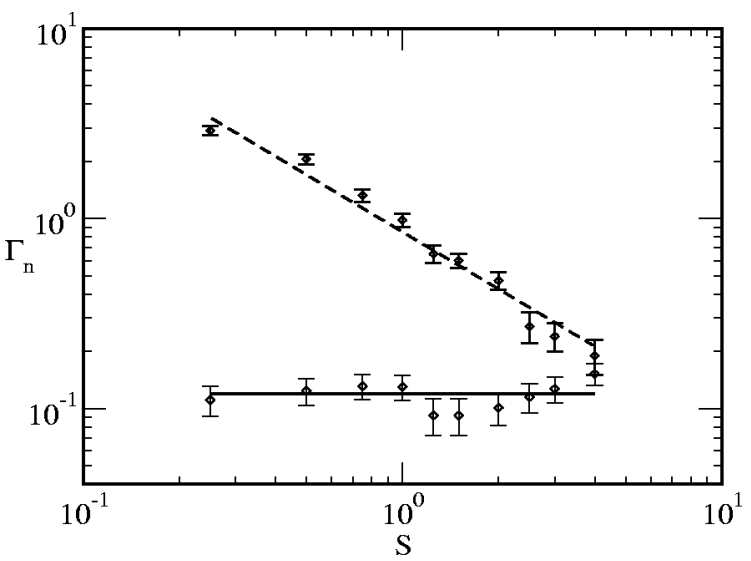

FIG. 2. Turbulence computations stopped around $t \approx 500$ lead to a feigned inverse dependence on shear $\hat{s}$ when averaged over the seemingly "saturated" phase. Only longer simulations show the high level of zonal flow spin up that is characteristic for a slab without curvature (lower points). The corresponding transport level is nearly independent from magnetic shear. havior, whereas the fully saturated state is dominated by a strong spin-up of zonal flows that suppresses turbulence to a low level nearly independent of shear.

\section{B. Magnetic field geometry}

The magnetic field geometry enters into the computations via metric elements and the curvature terms. The stellarator geometry we use is expressed in straight field line coordinates $(\psi, \theta, \zeta)$. The magnetic field in Clebsch form in terms of poloidal flux $2 \pi \psi$ and field line label $\alpha=\zeta-q \theta$ is $\mathbf{B}=\nabla \psi \times \nabla \alpha{ }^{18}$ Equivalently we can use $\xi \equiv \theta-\iota \zeta=-\iota \alpha$, where $\iota=1 / q$. It is $q=\partial \chi / \partial \psi$, and $\psi^{\prime}=\iota \chi^{\prime}$ where $2 \pi \chi$ is the toroidal flux. We define a radial coordinate $s$ with $\chi$ $=\left(\frac{1}{2}\right) B_{0} a_{0}^{2} s$. Then we have for the magnetic field the representation $\mathbf{B}=\nabla \chi \times \nabla \xi$. It holds that $\nabla \alpha=-q \nabla \xi-\xi \nabla q$ where $q=q(\chi)$.

The rescaled flux tube coordinate system used in DALF3 is expressed by the notation $x=\left(V-V_{0}\right) / V^{\prime}, y=-\psi V^{\prime} / L_{\|}$ and $z=\eta L_{\|}$. Here $L_{\|}=B_{0} / \psi_{V}^{\prime}=q R_{0}, V=s V_{0}$, and $V_{0}$ $=\left(2 \pi R_{0}\right)\left(a_{0}^{2} \pi\right)$. If the flux surfaces are not circular then $a_{0}$ and $R_{0}$ are the average minor and major plasma radius. The parallel coordinate $\eta$ may in principle be identified with either $\theta$ or $\zeta$. The magnetic field in this representation is

$$
\mathbf{B}=B_{0} \hat{\nabla} x \times \hat{\nabla} y .
$$

Expressing the reciprocal basis vectors in terms of $(s, \theta, \zeta)$ we thus get

$$
\begin{aligned}
& \nabla x=\frac{\partial r}{\partial V} \nabla V=\frac{1}{2 \sqrt{s}} \nabla s, \\
& \nabla y=-\frac{\psi_{V}^{\prime}}{B_{0}} \frac{1}{\partial r / \partial V} \nabla \alpha=\sqrt{s} \nabla \xi+\frac{\xi}{2 \sqrt{s}} \nabla s, \\
& \nabla z=\frac{B_{0}}{\psi_{V}^{\prime}} \nabla \theta=L_{\|} \nabla \eta .
\end{aligned}
$$

The relevant metric elements then are $g^{x x}=\left(\frac{1}{4} s\right) g^{s s}, g^{x y}$ $=\left(\frac{1}{2}\right) g^{s \xi}+(\xi / 4 s) g^{s s}$ and $g^{y y}=s \cdot g^{\xi \xi}$. In axisymmetric systems one can set $\xi \equiv 0$ without loss of generality. For flux tube representations of three-dimensional systems $\xi$ remains a free parameter.

Magnetic field line shear is generally defined as

$$
S=-\mathbf{B} \cdot \nabla \Lambda, \quad \text { where } \Lambda=g^{\psi \xi} / g^{\psi \psi} .
$$

Normal curvature is defined as $\kappa_{N}=\kappa \cdot \mathbf{N}$ and geodesic curvature as $\kappa_{G}=\kappa \cdot \mathbf{G}$ where the curvature vector is $\kappa$ $=\nabla \ln B$ for low-beta plasmas. The normal vector is $\mathbf{N}$ $=\nabla \psi$ and the geodesic vector is $\mathbf{G}=(\mathbf{B} \times \nabla \psi) /(\nabla \psi \cdot \nabla \chi)$ $=(\nabla \xi-\Lambda \nabla \psi)$. We note that the magnetic shear $S$ can also be expressed in terms of the geodesic vector as

$$
S=-q \mathbf{G} \cdot \nabla \times \mathbf{G} .
$$

\section{Curvilinear representation}

To express the derivative operators in terms of general curvilinear coordinates we write $u^{i}(\mathbf{R})=(s, \theta, \zeta)$ for $i$ $=(1,2,3)$. The tangent basis vectors of the coordinate curves 
defined by $u^{i}$ with respect to a Cartesian position vector $\mathbf{R}$ are given as $\mathbf{e}_{i}=\left(\partial \mathbf{R} / \partial u^{i}\right)$. The reciprocal basis vectors are $\mathbf{e}^{i}=\nabla u^{i}$. The notation used in the following employs the usual summation convention over equal indices $(i, j, k)$.

The Laplacian acting on a scalar $f$ in general curvilinear coordinates is

$$
\nabla^{2} f=\nabla \cdot(\nabla f)=\nabla \cdot\left(\mathbf{e}^{i} \frac{\partial f}{\partial u^{i}}\right)=\nabla \cdot\left(\mathbf{e}_{j} g^{i j} \frac{\partial f}{\partial u^{i}}\right) .
$$

We have expressed $\mathbf{e}^{i}$ as a linear combination of reciprocal basis vectors by writing $\mathbf{e}^{i}=\mathbf{e}_{j}\left(\mathbf{e}^{i} \cdot \mathbf{e}^{j}\right)$ and define $g^{i j} \equiv \mathbf{e}^{i} \cdot \mathbf{e}^{j}$. Expanded with the Jacobian $J \equiv \mathbf{e}_{1} \cdot \mathbf{e}_{2} \times \mathbf{e}_{3}=\left(\mathbf{e}^{1} \cdot \mathbf{e}^{2} \times \mathbf{e}^{3}\right)^{-1}$ we can write $\mathbf{e}_{j}=J\left(\mathbf{e}^{k} \times \mathbf{e}^{i}\right)$ and also, by use of the curl operator relations, $\mathbf{e}^{k} \times \mathbf{e}^{i}=\nabla \times\left(u^{k} \nabla u^{i}\right)$. This gives (considering $\nabla \cdot \nabla \times=0)$

$$
\nabla \cdot\left(\nabla \times\left[u^{k} \nabla u^{i}\right] J g^{i j} \frac{\partial}{\partial u^{i}} f\right)=\frac{1}{J} \mathbf{e}_{j} \cdot \nabla\left(J g^{i j} \frac{\partial}{\partial u^{i}} f\right) .
$$

By expressing $\nabla=\mathbf{e}^{j}\left(\partial / \partial u^{j}\right)$ and using $\mathbf{e}_{j} \cdot \mathbf{e}^{j}=1$ we may now write

$$
\nabla^{2} f=\frac{1}{J} \frac{\partial}{\partial u^{j}}\left(J g^{i j} \frac{\partial}{\partial u^{i}}\right)
$$

We can separate the variation of the Jacobian by writing:

$$
\nabla^{2} f=g^{i j} \frac{\partial^{2}}{\partial u^{i} \partial u^{j}} f+\frac{1}{J}\left(\frac{\partial f}{\partial u^{i}}\right)\left(\frac{\partial}{\partial u^{j}} J g^{i j}\right) \equiv \nabla_{0}^{2} f+\nabla_{1}^{2} f .
$$

The perpendicular Laplacian is found in general using the perpendicular metric elements in this expression, $g_{\perp}^{i j}=g^{i j}$ $-b^{i} b^{j}$, but here the flute mode ordering enters to simplify the matter. The flute-like character of drift wave turbulence implies $k_{\|} \ll k_{\perp}$ and thus $\partial / \partial z \ll \partial / \partial x \sim \partial / \partial y$ for a flux tube coordinate system $u^{i}=(x, y, z)$ in drift ordering. For the perpendicular direction in $(x, y)$ the ordering assumes amplitudes in proportion to their scale, so that terms containing second derivatives $\left(\partial^{2} / \partial u^{i} \partial u^{j}\right)$ of fluctuations appear much larger than terms including only first derivatives $\left(\partial / \partial u^{i}\right)$. Within drift ordering we can thus neglect the $\nabla_{1}^{2}$ term against the $\nabla_{0}^{2}$ term, as well as the components of $g$ involving the component for which $b^{i}$ is nonvanishing (here, $z$ ). The perpendicular Laplacian then does not depend explicitly on the Jacobian, and in both Hamada and Boozer coordinates it takes the same simple form

$$
\nabla_{\perp}^{2}=g^{x x} \frac{\partial^{2}}{\partial x^{2}}+2 g^{x y} \frac{\partial^{2}}{\partial x \partial y}+g^{y y} \frac{\partial^{2}}{\partial y^{2}} .
$$

It is important to note, however, that the variation of $B^{2}$ around the flux surface still enters the nonlinear vorticity through $B^{-2} \nabla_{\perp}^{2}$, through the dependence of $B^{2}$ upon the parallel coordinate, $z$, although solely as a $z$-dependent multiplier within each $x y$-plane. This has been found to have strong impact upon the strength of drift wave turbulence. ${ }^{19}$

The gradient parallel to a field line is defined as $\nabla_{\|} f=\mathbf{b}$ - $\nabla f=(\mathbf{B} / B) \cdot \nabla f$. We start with the general form for the gra-

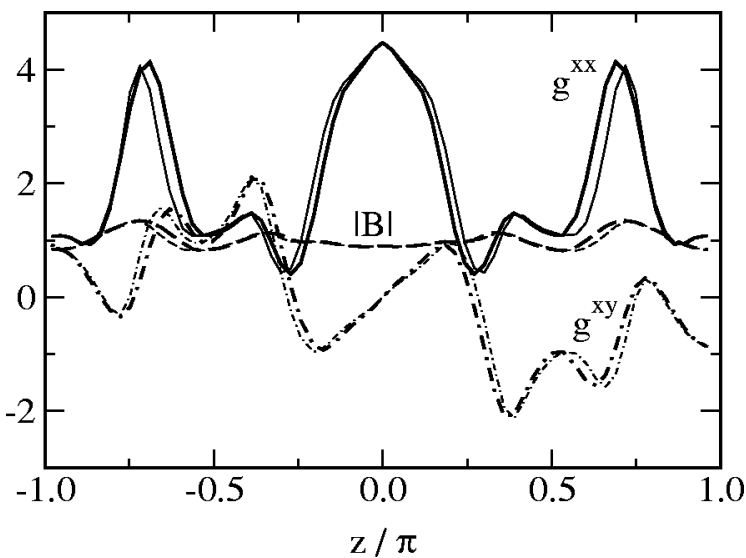

FIG. 3. Comparison of Boozer coordinate representation (bold lines) and Hamada coordinates (thin lines) for the quantities $g^{x x}, g^{x y}$, and $|B|$ of the stellarator W7-X $(\xi=0)$.

dient, $\nabla f=\mathbf{e}^{i}\left(\partial / \partial u^{i}\right) f$, and write the magnetic field in Clebsch form $\mathbf{B}=\nabla \psi \times \nabla y=\psi^{\prime} \nabla x \times \nabla y$. Therefore, $b^{x}=\mathbf{B}$ $\cdot \nabla x=0$ and $b^{y}=\mathbf{B} \cdot \nabla y=0$ and

$$
\nabla_{\|} f=b^{z} \frac{\partial}{\partial z} f .
$$

Here $b^{z}=(\mathbf{B} / B) \cdot \nabla z=\left(\psi^{\prime} / B J\right)$.

Some of the parallel derivative terms are actually divergences:

$$
\nabla_{\|} \widetilde{j}_{\|}=\nabla \cdot\left(\widetilde{j}_{\|} \mathbf{b}\right)=\nabla \cdot\left(\frac{\widetilde{j}_{\|} \mathbf{B}}{B}\right) .
$$

Expressing $\mathbf{b}$ in curvilinear coordinates we find

$$
\nabla_{\|} \tilde{j}_{\|}=\nabla \cdot\left[\left(\tilde{j}_{\|} b^{i}\right) \mathbf{e}_{i}\right]=\frac{1}{J} \frac{\partial}{\partial u^{i}}\left[J\left(\tilde{j}_{\|} b^{i}\right)\right] .
$$

The second derivative in parallel direction is $\nabla_{\|}^{2} f=\nabla$ bb $\cdot \nabla f=\nabla \cdot\left(\mathbf{b} \nabla_{\|}\right) f$. In the same fashion as above we find

$$
\nabla_{\|}^{2} f=\frac{1}{J} \frac{\partial}{\partial z}\left(J b^{z} \nabla_{\|}\right) f .
$$

The implementation of Boozer coordinates into the DALF turbulence codes instead of the unit-Jacobian Hamada coordinates used previously requires the Jacobian $J$ to be stored as an additional quantity as a function of the parallel flux tube coordinate $z$. We note that for large aspect ratio configurations like W7-X the difference between Boozer and Hamada coordinates is qualitatively negligible, as shown in Fig. 3. For lower aspect ratios, as present in most tokamaks, retention of the Jacobian's dependence in these parallel divergence forms is required.

\section{HOW TO MODEL A STELLARATOR}

\section{A. Wendelstein 7-X and H1-NF}

Helical Advanced Stellarator (Helias) configurations ${ }^{20}$ are based on the ideas of A. Schlüter for improving the confinement and equilibrium properties of a classical stellarator. The first experiment in this advanced line was the partially optimized stellarator Wendelstein $7-\mathrm{AS}^{21}{ }^{21}$ that was in opera- 
tion for 14 years until 2002, and was able to succesfully demonstrate the feasibility of the concept. As the next step (currently under construction), Wendelstein 7-X has been developed $^{7}$ to test the reactor relevance of Helias configurations. The basic properties of a Helias configuration are a magnetohydrodynamic (MHD) stable plasma with temperature $T$ and density $n$ up to a pressure, averaged over the whole plasma, of $\langle\beta\rangle=\left\langle 2 \mu_{0} n T / B^{2}\right\rangle \approx 5 \%$ with low Shafranov shift due to reduction of Pfirsch-Schlüter currents, and low neoclassical losses. ${ }^{22}$ Further criteria for optimization are good confinement properties for $\alpha$-particles, low bootstrap currents and well-defined magnetic surfaces of the vacuum field without major resonances. ${ }^{23,24} \mathrm{~W} 7-\mathrm{X}$ will be realized as a five field-period configuration with rotational transform $\iota$ close to unity and low global shear.

A Helical Axis Stellarator (Heliac) achieves rotational transform by combining the poloidal field produced by an axisymmetric currrent-carrying ring with the field produced by helically staggered toroidal field coils. ${ }^{25}$ The Heliac H1NF, the Australian national plasma fusion research facility, is a three-period configuration designed with flexibility in the magnetic field in order to gain a detailed understanding of the behavior of Heliac plasmas. ${ }^{8}$ Relatively low plasma density allows access to fluctuation measurements by probes, that has recently led to a first direct observation of zonal flow like structures. ${ }^{26}$

The equilibrium configurations of W7-X and H1-NF were obtained with the code $\mathrm{NEMEC}^{27}$ and tabulated as a Fourier series for magnetic field strength $|B|$ and cylinder coordinates $\mathbf{X}=(R, \varphi, Z)$ in terms of flux coordinates $\mathbf{u}$ $=\left(u^{1}, u^{2}, u^{3}\right)=(s, \theta, \zeta)$. From these, covariant basis vectors $\mathbf{e}_{i}=\partial \mathbf{X} / \partial u^{i}$ and in consequence the contravariant vectors $\mathbf{e}^{i}$ $=\nabla u^{i}=\mathbf{e}_{j} \times \mathbf{e}_{k} /\left(\mathbf{e}_{i} \cdot \mathbf{e}_{j} \times \mathbf{e}_{k}\right)$ are obtained. The resulting Jacobian, metric elements $g^{i j}$ and derivatives of $|B|$ (i.e., curvature) then describe the magnetic field geometry.

\section{B. Flux tube models of a stellarator}

An extension of the globally consistent flux tube model in DALF3 ${ }^{12}$ from an axisymmetric tokamak to a stellarator is in principle straightforward, but not without fundamental obstacles. When in general the rotational transform $\iota$ is irrational, the flux tube will not close in on itself. The code DALF3 solves this problem by aligning the flux tube in a local approximation centrally around a rational field line and corrects deformation in the perpendicular plane caused by finite shear with an appropriate shear shift transformation. ${ }^{13}$ In a tokamak, this approach is appropriate even for a high order rational ratio $\iota=m / n$, as the geometric background properties only depend on the poloidal angle $\theta$, regardless of the number of toroidal circuits until field line closure.

In a stellarator, however, the axisymmetry is broken and helical variations of the metric become significant. The variations of background geometry along the field line scale with the number of toroidal circuits and field periods $N_{f}$, and so does the number of necessary parallel grid points. For small rotational transform, the number of grid points evidently diverges rapidly. Thus, for future quantitative simulations of stellarator turbulence, a 3D hollow flux cylinder

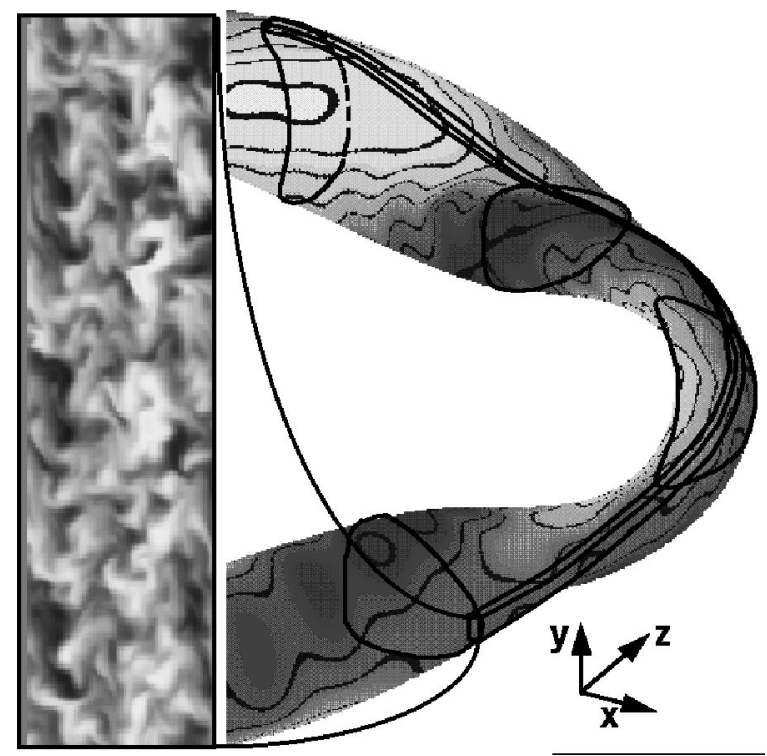

FIG. 4. Flux tube aligned at the edge of a stellarator (W7-X), and a poloidal cut in the computational $(x, y)$ plane showing density perturbations in driftAlfvén turbulence.

model, with full $y$-dependence of the metric, will be required. For our current purpose of qualitative identification of the relevant geometrical quantities that determine drift wave turbulence in stellarators, we can take advantage of the fact that the edge rotational transform of advanced stellarators such as W7-X and H1-NF is near unity: $\iota_{W 7-X}\left(a_{0}\right)$ $=0.98 \approx 1$ on the last closed flux surface with $r=a_{0}$. Of course, the surface with $\iota \equiv 1$ would be governed by generic island formation of the five-period Helias. When we assume a "rotational transform" of unity, we here mean a toroidal periodicity in the magnetic field structure for the computation of geometric quantities. So although we do not map the geometrical properties of W7-X, H1-NF, or any other specific configuration exactly, we still gain a quite accurate model of Helias type geometry. The original, exact value of the rotational transform on the flux surface of choice is still used for field line connection and shear shift properties in the turbulence computations.

In contrast to the axisymmetric case, it is also no longer true that one field line is representative for the whole flux surface. For $N$-fold toroidal symmetry, the flux tubes labeled $\xi=\theta-\iota \zeta=i / N$ (with $\xi \in[0 \ldots 1]$ ) are identical for $i$ $\in[0,1, \ldots, N]$. To cover a representative proportion of the flux surface, simulations on four different field lines for $\xi$ $\in[0 \ldots 1 / N]$ with $N=5$ were usually found to be sufficient. In an earlier study of drift wave turbulence in stellarator geometry presented in Ref. 5, a strong quantitative variation of transport amplitude was found with $\xi$ of around a factor of two. For the qualitative studies of the present work we keep $\xi=0$ constant. The principal arguments concerning properties of the parallel structure (although spatially differing) and zonal flow formation do not depend on a specific choice of the flux tube label. A section of W7-X showing the location of a flux tube at the plasma edge is shown combined with a poloidal cut view of simulated density fluctuation structures in Fig. 4. 


\section{TURBULENCE STRUCTURES IN THE PLASMA EDGE}

Drift wave turbulence driven by the free energy from thermal gradients self-organizes into various nonlinear structures. For the present work we limit our attention to cold-ion drift-Alfvén wave turbulence in the plasma edge for a specified, "typical" set of plasma parameters. Changes in turbulence structures that arise for variations in these parameters, for example, an additional temperature gradient, the presence of a population of trapped electrons, or a shift to more MHD ballooning-like character are thus not regarded here. We will rather focus on the influence of magnetic field geometry and plasma shaping on the spatial and temporal arrangement of fluctuating quantities.

The basic constituents of drift wave edge turbulence are fluid-like vortices in the drift plane perpendicular to the magnetic field direction, where the convection is maintained by the $\mathbf{E} \times \mathbf{B}$ velocity. The eddies of largest amplitude in the mode spectrum have spatial scales of a few $\rho_{s}$. The nonlinearities involving the pressure (and electron inertia and sound waves) transfer energy from one spectral range to another by direct cascade of basically three-wave interactions, limited by a dissipation range at high wavenumbers (high$k_{\perp}$ ). The vorticity nonlinearity has a dual cascade tendency sending enstrophy preferentially to small scales but energy to larger ones, ${ }^{28,29}$ reducing the amount of high- $k_{\perp}$ dissipation for a given level of nonlinear activity. ${ }^{9}$ This allows for nonlinear instability by widening the range of available phase shifts between Fourier components of the pressure and electrostatic potential. ${ }^{4}$

Linear analysis of drift modes (as, for example, in Ref. 30) would suggest that the largest growth rates occur mostly for zero radial mode number $\left(k_{x}=0, k_{y} \approx 0.3\right)$ where modes tended to form elongated structures ("streamers") in the radial direction. In the associated turbulence, however, the nonlinear interactions do not favor well pronounced streamers. Both three-wave nonlinear decorrelation and interaction with radially sheared flows in the drift direction, generated by the vorticity nonlinearity, are responsible. The inverse cascade tendency leads to transfer of eddy energy into $k_{y}$ $=0$ modes with radial extension comparable to the size of the turbulence $\left(k_{x} \approx 0.3\right)$, causing a rigid poloidal rotation of the entire flux surfaces. These "zonal flows" do not participate in cross field transport, but act back on the turbulence as a sink for $\mathbf{E} \times \mathbf{B}$ energy, ${ }^{31,32}$ and as a decorrelation mechanism for vortices and streamers. ${ }^{33}$ The geodesic curvature operator $\mathcal{K}^{x} \partial_{x}$ couples the zonal flow mode indirectly back to the turbulence. ${ }^{15}$ The saturated fluctuation and transport levels in edge and core drift wave turbulence are strongly regulated by formation and sustainment of radially sheared zonal flow structures.

The disturbances in the electric field $\mathbf{E}$ implied by the presence of the $\mathbf{E} \times \mathbf{B}$ eddies are caused by the action of the polarization current (ion inertia) to balance all the other currents so that the charge density remains small. In drift wave turbulence the parallel current is the most important of these, ${ }^{10}$ while for ballooning or interchange modes the diamagnetic current is strongest. ${ }^{34}$ When drift wave conditions predominate, the electron pressure and electrostatic potential are coupled through parallel currents, with departures from the static force balance mediated primarily through electromagnetic induction and resistive friction, but also the electron inertia, which is not negligible. The strong parallel dynamics causes the modes to become flute-like and greatly extended in the parallel direction $\left(k_{\|} \ll k_{x}\right)$. In the presence of magnetic shear, the perpendicular vortices become twisted with the field lines and are finally, nonlinearly, torn apart to smaller scales. Parallel structures in drift wave turbulence originate from inhomogeneities of the background geometry; specifically, from an (unfavorable) normal component of magnetic curvature serving catalytic functions or as ballooning drive mechanism (depending on plasma parameters), and through an effective magnetic shear length $L_{s}$ either extending over the whole flux tube domain or in localized regions along $z$. Both conditions can affect the damping and drive of fluctuations, and therefore, contribute to the regulation of turbulent transport levels across flux surfaces.

Summarizing, nonlinear structure formation in drift wave edge turbulence can be observed in any of the flux tube directions: Radially sheared zonal flows have finite extension in $x$, and can significantly reduce transport, the intensity of which depends on the background magnetic field geometry. The structures in perpendicular drift direction $y$ are governed by the turbulence spectrum with maximum amplitude for scales of $k_{y} \sim 0.3$, emergence of $k_{y}=0$ flows and a selfsimilar cascade region for higher wave numbers up to dissipation ranges at very small scales. The parallel mode structure can serve as an indicator of ballooning character of the turbulence and of effective quenching by local magnetic field line shear. All of the above phenomena depend on time at fluctuation scales but retain their specific characteristics over time averages.

\section{A. Parallel mode structure}

Linear theory of cold ion drift waves in a local ballooning model for Helias geometry suggests that the parallel structure of the mode is governed by a combination of helical and toroidal ballooning. ${ }^{30}$ We now compute nonlinear turbulent drift-Alfvén modes in a Helias by applying the stellarator flux tube approximation, as described in Sec. IV B, to the Wendelstein 7-X geometry. In contrast to linear modelling, no helical ballooning is observed in the turbulent state for realistic edge parameters. The drift wave structure limits itself to long parallel wavelengths, which average over the magnetic structure.

We note especially a lack of asymmetry along the field line when the normal curvature departs from the simple sinusoidal form of a tokamak. Figure 5 shows minor variations in the turbulence amplitudes $\widetilde{\phi}$ and $\widetilde{n}$ along the field line direction $z$. The structure follows more from the local shear than from the normal curvature, as can be seen from Fig. 6. These results highlight the role of local magnetic shear in stellarator turbulence and structure formation, in addition to the known damping effects that we studied previously. 6,35 Specifically, the amplitude of $\Gamma_{n}(z)$, averaged over time and $(x, y)$, is distinctly although not dramatically reduced at regions of large local shear. (Here the flux tube with $\xi=0.5$ was used 


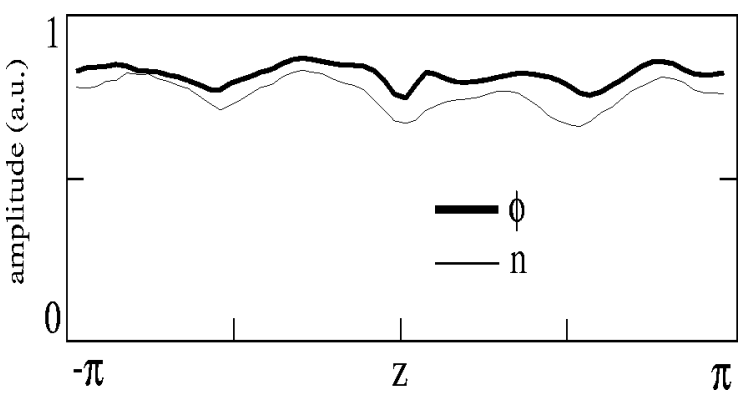

FIG. 5. Turbulence mode structure in a flux tube along $z$ of potential and density fluctuations averaged over the perpendicular $(x, y)$ plane (top) in W7-X (here $\xi=0.5)$.

with $z=0$ at the triangular outboard plane because of its more pronounced local shear structure.)

\section{B. Radial zonal flow structure}

As an intrinsic part of the turbulence dynamics zonal $E$ $\times B$ flows are generated via Reynold stress coupling of eddies to flows with both $k_{y}=0$ and $k_{\|}=0$. By a decorrelation of vortices and transfer of energy from eddies to the flow the turbulence amplitude is reduced. For tokamak geometry the suppression effects have been shown to be a relevant part of the overall dynamics. ${ }^{33}$

When zonal flows are switched off in computations by setting the flux surface average Reynolds stress $\mathbf{v}_{E} \cdot \nabla \nabla_{\perp}^{2} \phi$ to zero, the level of transport was found to be higher by a factor of 2-5 in toroidal geometry. ${ }^{36}$ For Wendelstein $7-X$ stellarator geometry $(\xi=0)$ and otherwise same parameters as given in Ref. 36, the damping effects are also clearly demonstrated in Fig. 7 over a range of various levels of collisionality $\hat{\nu}$. The suppression factor of around 5-8 is between the previous tokamak result including geodesic curvature, and the large amplitude flow case of a sheared slab without the curvature effects.

The geodesic curvature, $\mathcal{K}^{x} \partial_{x}$, couples the zonal flow mode over sideband pressure perturbations as a loss channel back to the turbulence. ${ }^{15}$ The zonal flow dynamics, therefore, gives the turbulence an overall different character in toroidal geometry. A reduction of geodesic curvature is a specific design criterion for Helias stellarators, and, combined with the lowering effect of a larger aspect ratio $(A \approx 10)$ on curvature, the damping of flows is reduced in comparison to a

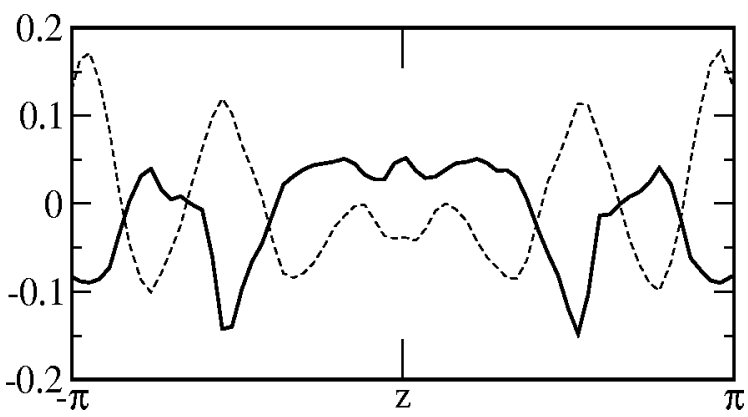

FIG. 6. Structure of $|B|$ (dotted line) and $\kappa_{N}$ (solid line) in the flux tube of Fig. 5 along the parallel coordinate $z$.

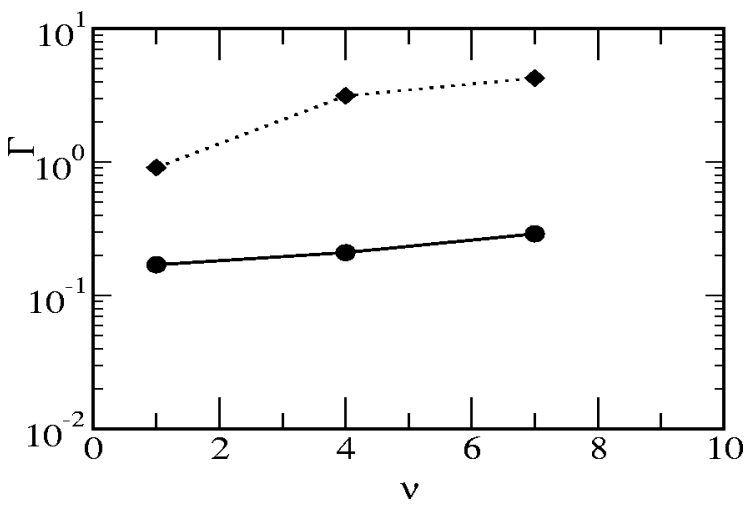

FIG. 7. The nominal transport $\Gamma_{n}$ (dark line) for various collisionalities $\hat{v}$ in W7-Xstellarator geometry is effectively lowered in comparison to the case with the flux surface averaged vorticity set to zero (dotted line). Zonal flows are, similar to the tokamak case, an essential part of the turbulence dynamics.

tokamak $(A \approx 3)$ with otherwise similar plasma parameters. A complementary path to transport optimization than the one taken by helical advanced stellarators is the design of quasiaxisymmetric (QA) configurations. The aspect ratio of QA stellarators is low and comparable to that of a tokamak, which might seem unfavorable from the point of view of zonal flow generation presented above. Yet one may expect that the reduced helical ripple helps to maintain low viscous damping of toroidal rotation, similarly to a tokamak, and thus also favor the generation of sheared turbulence reducing flows. $^{37}$

Figure 8 shows the time evolution of turbulent transport $\Gamma_{n}$ in the edge geometry of Heliac H1-NF for typical parameters $\hat{\mu}=4, \hat{\nu}=1.5, \beta=4$, and $\varepsilon=12000$. The turbulence transport amplitudes saturate rapidly when the zonal flows are removed from the computations (upper curve) on a high level. Including the complete dynamics with zonal flows the transport level is again reduced significantly and shows in-

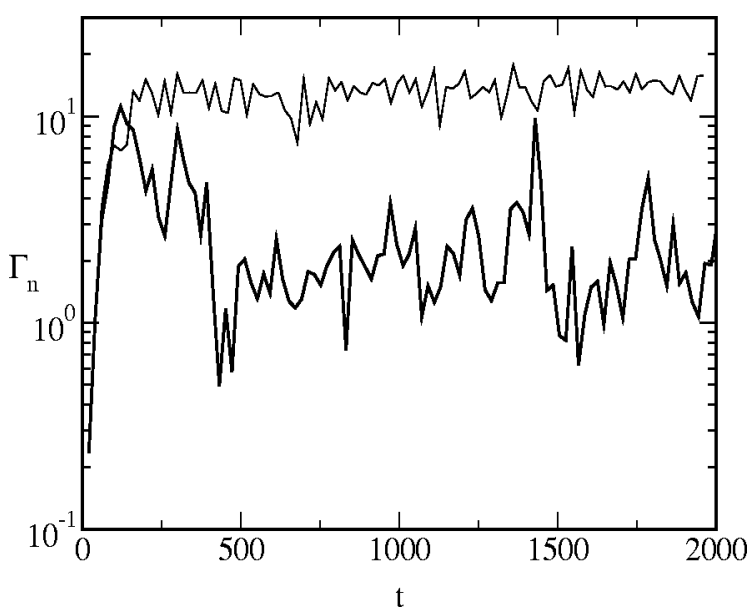

FIG. 8. Time evolution in the first 2000 numerical steps (output data after every 20 steps) of $E \times B$ transport $\Gamma_{n}$ in a turbulence computation in H1-NF stellarator geometry. Transport is lowered when the full dynamics is included (lower curve) compared to the case without zonal flows. Intermittency in the transport dynamics is caused by a rearrangement of zonal structures that emerge after $t \approx 400$. 


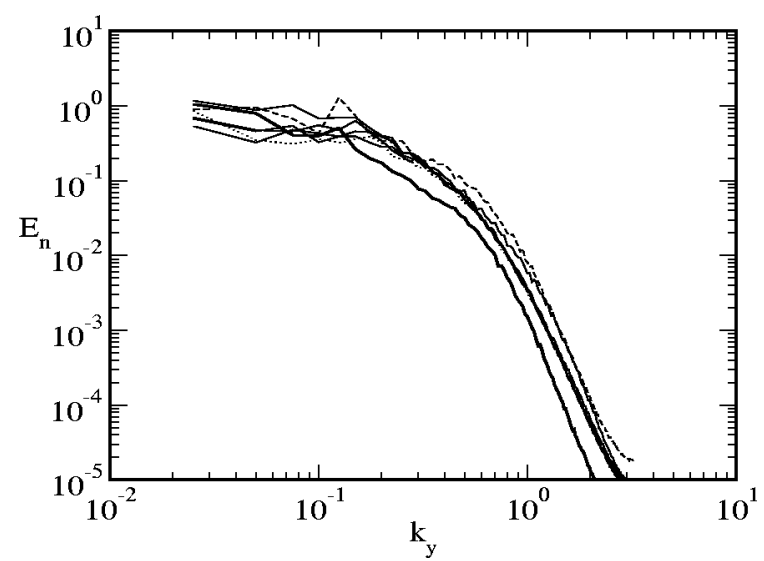

FIG. 9. Spectra of $k_{y}$ obtained by simulations in various stellarator geometries and simple tokamak models show a high degree of similarity that indicates universality in the perpendicular structural character of drift wave turbulence independent of the specific field configuration.

termittent behavior at all times, which results mainly from a rearrangement of fluctuating zonal flow structures and amplitudes.

\section{Perpendicular mode structure}

A comparison of the density fluctuation spectra $E_{n}\left(k_{y}\right)$ between various geometries in Fig. 9 shows a high degree of similarity. The spectra shown were obtained from simulations using different stellarator geometries and simple tokamak models. ${ }^{5}$ The good interconfigurational agreement reproduces an empirical similarity for measured fluctuations in different experimental devices as found by Pedrosa et al. ${ }^{38} \mathrm{In}$ their case frequency spectra were compared after introduction of a device specific, frequency dependent scaling factor called $\lambda$. From our drift-Alfvén turbulence simulations we conclude that this scaling factor is independent of the geometry of the different experiments. As other causes for the variation of Pedrosa's $\lambda$ we rather suggest device specific plasma parameters and differences in background velocity shear profiles. These have not been included in our simulations.

\section{CONCLUSIONS}

We have shown computations of drift-Alfvén wave turbulence in the edge of simple tokamak models and two stellarators, the Helias type W7-X and the Heliac type H1-NF. The approximation procedure to include three-dimensional magnetic field configurations into flux tube models of the geometry has been outlined. Turbulent structure formation in terms of parallel and perpendicular mode and radial zonal flow structures has been discussed. Specific characteristics of these stellarators in comparison to (lower aspect ratio) circular tokamaks are a less pronounced ballooning structure of the modes, a strong influence of local magnetic shear on amplitude structure and average, and stronger level of zonal flows due to lower geodesic curvature. Although the drift wave character of fluctuations is preserved in all simulations, the turbulent transport shows considerably different levels, depending strongly on the various discussed geometric aspects of the magnetic field configuration. A stellarator configuration is thus in principle also amenable to optimization with respect to turbulent transport properties.

\section{ACKNOWLEDGMENTS}

We thank E. Strumberger (IPP Garching) and B. McMillan (ANU Canberra) for supply of the numerical stellarator equilibrium data.

This work was partly funded by grants within the "Australian-German Joint Research Co-operation scheme" (PPP project no. D/0205403).

${ }^{1}$ U. Stroth, Plasma Phys. Controlled Fusion 40, 9 (1998).

${ }^{2}$ C. Hidalgo, Plasma Phys. Controlled Fusion 37, A53 (1995).

${ }^{3}$ M. Wakatani and A. Hasegawa, Phys. Fluids 27, 611 (1984).

${ }^{4}$ B. D. Scott, Phys. Fluids B 4, 2468 (1992).

${ }^{5}$ A. Kendl, B. D. Scott, and W. Wobig, Plasma Phys. Controlled Fusion 42, L23 (2000).

${ }^{6}$ A. Kendl and B. D. Scott, Phys. Rev. Lett. 90, 035006 (2003).

${ }^{7}$ C. Beidler, G. Grieger, F. Herrnegger et al., Fusion Technol. 17, 148 (1990).

${ }^{8}$ S. M. Hamberger, B. D. Blackwell, L. E. Sharp, and B. D. Shenton, Fusion Technol. 17, 123 (1990).

${ }^{9}$ B. D. Scott, New J. Phys. 4, 52 (2002).

${ }^{10}$ B. D. Scott, Plasma Phys. Controlled Fusion 39, 1635 (1997).

${ }^{11}$ F. L. Hinton and C. W. Horton, Jr., Phys. Fluids 14, 116 (1971).

${ }^{12}$ B. D. Scott, Phys. Plasmas 5, 2334 (1998).

${ }^{13}$ B. D. Scott, Phys. Plasmas 8, 447 (2001).

${ }^{14}$ S. I. Braginskii, Rev. Plasma Phys. 1, 205 (1965).

${ }^{15}$ B. D. Scott, "Zonal flows and electromagnetic drift wave turbulence," Phys. Lett. A (submitted).

${ }^{16}$ S.-T. Tsai, F. W. Perkins, and T. H. Stix, Phys. Fluids 13, 2108 (1970).

${ }^{17}$ W. Dorland and G. Hammett, Phys. Fluids B 5, 812 (1993).

${ }^{18}$ R. L. Dewar and A. H. Glasser, Phys. Fluids 26, 3038 (1983).

${ }^{19}$ B. D. Scott, Plasma Phys. Controlled Fusion 39, 471 (1997).

${ }^{20}$ J. Nührenberg and R. Zille, Phys. Lett. A 114, 129 (1986).

${ }^{21} \mathrm{H}$. Renner, W7AS Team, NBI Group, ICF Group, and ECRH Group, Plasma Phys. Controlled Fusion 31, 1579 (1989).

${ }^{22}$ W. Lotz, J. Nührenberg, and C. Schwab, Plasma Phys. Controlled Nucl. Fusion Res. 2, 603 (1991).

${ }^{23}$ H. Wobig, Plasma Phys. Controlled Fusion 41, A159 (1999).

${ }^{24}$ A. H. Boozer, Plasma Phys. Rep. 23, 483 (1997).

${ }^{25}$ A. H. Boozer, T. K. Chu, R. L. Dewar et al., Plasma Phys. Controlled Nucl. Fusion Res. 3, 129 (1983).

${ }^{26}$ M. G. Shats and W. M. Solomon, Phys. Rev. Lett. 88, 045001 (2002).

${ }^{27}$ P. S. Hirshman et al., Comput. Phys. Commun. 43, 143 (1986).

${ }^{28}$ F. Y. Gang, B. D. Scott, and P. H. Diamond, Phys. Fluids B 1, 1331 (1989).

${ }^{29}$ B. D. Scott, H. Biglari, P. W. Terry, and P. H. Diamond, Phys. Fluids B 3, 51 (1991).

${ }^{30}$ A. Kendl and H. Wobig, Phys. Plasmas 6, 4714 (1999).

${ }^{31}$ P. Diamond and Y. Kim, Phys. Fluids B 3, 1626 (1991).

${ }^{32}$ B. D. Scott, Plasma Phys. Controlled Fusion 34, 1977 (1992).

${ }^{33}$ T. S. Hahm, M. A. Beer, Z. Lin, G. W. Hammett, W. W. Lee, and W. M. Tang, Phys. Plasmas 6, 922 (1999).

${ }^{34}$ B. N. Rogers and J. F. Drake, Phys. Rev. Lett. 79, 229 (1997)

${ }^{35}$ A. Kendl, Plasma Phys. Controlled Fusion 43, 1559 (2001).

${ }^{36}$ B. D. Scott, F. Jenko, and A. Kendl, Proceedings of the 18th IAEA Fusion Energy Conference, Sorrento, 2000 (IAEA-CN-77, International Atomic Energy Association, Vienna, 2001), THP1/12.

${ }^{37}$ M. C. Zarnstorff, L. A. Berry, A. Brooks et al., Plasma Phys. Controlled Fusion 43, A237 (2001).

${ }^{38}$ M. A. Pedrosa, C. Hidalgo, B. A. Carreras et al., Phys. Rev. Lett. 82, 3621 (1999). 